\title{
ANNALES
}

UNIVERSITATIS MARIAE CURIE-SKŁODOWSKA

LUBLIN - P OLONIA

\section{ALBO CARLOS CAVALHEIRO}

\section{An existence and approximation theorem for solutions of degenerate nonlinear elliptic equations}

\begin{abstract}
The main result establishes that a weak solution of degenerate nonlinear elliptic equations can be approximated by a sequence of solutions for non-degenerate nonlinear elliptic equations.
\end{abstract}

1. Introduction. Let $L$ be a degenerate elliptic operator in divergence form

$$
L u(x)=-\sum_{i, j=1}^{n} D_{j}\left(a_{i j}(x) D_{i} u(x)\right), D_{j}=\frac{\partial}{\partial x_{j}},
$$

where the coefficients $a_{i j}$ are measurable, real-valued functions whose coefficient matrix $A(x)=\left(a_{i j}(x)\right)$ is symmetric and satisfies the degenerate ellipticity condition

$$
\lambda|\xi|^{2} \omega(x) \leq \sum_{i, j=1}^{n} a_{i j}(x) \xi_{i} \xi_{j} \leq \Lambda|\xi|^{2} \omega(x),
$$

for all $\xi \in \mathbb{R}^{n}$ and almost everywhere $x \in \Omega \subset \mathbb{R}^{n}$ a bounded open set, $\omega$ is a weight function, $\lambda$ and $\Lambda$ are positive constants.

2010 Mathematics Subject Classification. 35J61, 35J70, 35D30.

Key words and phrases. Degenerate nonlinear elliptic equations, weighted Sobolev spaces. 
The main purpose of this paper (see Theorem 1.2) is to establish that a weak solution $u \in W_{0}^{1,2}(\Omega, \omega)$ for the nonlinear Dirichlet problem

$$
(P)\left\{\begin{array}{l}
L u(x)+g(u(x)) \omega(x)=f_{0}(x)-\sum_{j=1}^{n} D_{j} f_{j}(x) \text { in } \Omega, \\
u(x)=0 \text { on } \partial \Omega,
\end{array}\right.
$$

can be approximated by a sequence of solutions of non-degenerate nonlinear elliptic equations.

By a weight, we shall mean a locally integrable function $\omega$ on $\mathbb{R}^{n}$ such that $\omega(x)>0$ for a.e. $x \in \mathbb{R}^{n}$. Every weight $\omega$ gives rise to a measure on the measurable subsets on $\mathbb{R}^{n}$ through integration. This measure will be denoted by $\mu$. Thus, $\mu(E)=\int_{E} \omega(x) d x$ for measurable sets $E \subset \mathbb{R}^{n}$.

In general, the Sobolev spaces $\mathrm{W}^{k, p}(\Omega)$ without weights occur as spaces of solutions for elliptic and parabolic partial differential equations. For degenerate partial differential equations, i.e., equations with various types of singularities in the coefficients, it is natural to look for solutions in weighted Sobolev spaces (see [1]-[5], [8] and [10]).

A class of weights, which is particularly well understood, is the class of $A_{p}$-weights (or Muckenhoupt class) that was introduced by B. Muckenhoupt (see [7]). These classes have found many useful applications in harmonic analysis (see [9]). Another reason for studying $A_{p}$-weights is the fact that powers of the distance to submanifolds of $\mathbb{R}^{n}$ often belong to $A_{p}$ (see [6]). There are, in fact, many interesting examples of weights (see [5] for padmissible weights).

The following lemma can be proved in exactly the same way as Lemma 2.1 in [3] (see also, Lemma 3.1 and Lemma 4.13 in [1]). Our lemma provides a general approximation theorem for $A_{p}$ weights $(1 \leq p<\infty)$ by means of weights which are bounded away from 0 and infinity and whose $A_{p^{-}}$ constants depend only on the $A_{p}$-constant of $\omega$. Lemma 1.1 is the key point for Theorem 1.2, and the crucial point consists of showing that a weak limit of a sequence of solutions of approximate problems is in fact a solution of the original problem.

Lemma 1.1. Let $\alpha, \beta>1$ be given and let $\omega \in A_{p}(1 \leq p<\infty)$, with $A_{p}$-constant $C(\omega, p)$ and let $a_{i j}=a_{j i}$ be measurable, real-valued functions satisfying

$$
\lambda \omega(x)|\xi|^{2} \leq \sum_{i, j=1}^{n} a_{i j}(x) \xi_{i} \xi_{j} \leq \Lambda \omega(x)|\xi|^{2},
$$

for all $\xi \in \mathbb{R}^{n}$ and a.e. $x \in \Omega$. Then there exist weights $\omega_{\alpha \beta} \geq 0$ a.e. and measurable real-valued functions $a_{i j}^{\alpha \beta}$ such that the following conditions are met.

(i) $c_{1}(1 / \beta) \leq \omega_{\alpha \beta} \leq c_{2} \alpha$ in $\Omega$, where $c_{1}$ and $c_{2}$ depend only on $\omega$ and $\Omega$. 
(ii) There exist weights $\tilde{\omega}_{1}$ and $\tilde{\omega}_{2}$ such that $\tilde{\omega}_{1} \leq \omega_{\alpha \beta} \leq \tilde{\omega}_{2}$, where $\tilde{\omega}_{i} \in A_{p}$ and $C\left(\tilde{\omega}_{i}, p\right)$ depends only on $C(\omega, p)(i=1,2)$.

(iii) $\omega_{\alpha \beta} \in A_{p}$, with constant $C\left(\omega_{\alpha \beta}, p\right)$ depending only on $C(\omega, p)$ uniformly on $\alpha$ and $\beta$.

(iv) There exists a closed set $F_{\alpha \beta}$ such that $\omega_{\alpha \beta} \equiv \omega$ in $F_{\alpha \beta}$ and $\omega_{\alpha \beta} \sim$ $\tilde{\omega}_{1} \sim \tilde{\omega}_{2}$ in $F_{\alpha \beta}$ with equivalence constants depending on $\alpha$ and $\beta$ (i.e., there are positive constants $c_{\alpha \beta}$ and $C_{\alpha \beta}$ such that $c_{\alpha \beta} \tilde{\omega}_{i} \leq \omega_{\alpha \beta} \leq C_{\alpha \beta} \tilde{\omega}_{i}$, $i=1,2)$. Moreover, $F_{\alpha \beta} \subset F_{\alpha^{\prime} \beta^{\prime}}$ if $\alpha \leq \alpha^{\prime}, \beta \leq \beta^{\prime}$, and the complement of $\bigcup_{\alpha, \beta>1} F_{\alpha \beta}$ has zero measure.

(v) $\omega_{\alpha \beta} \rightarrow \omega$ a.e. in $\mathbb{R}^{n}$ as $\alpha, \beta \rightarrow \infty$.

(vi) $\lambda \omega_{\alpha \beta}(x)|\xi|^{2} \leq \sum_{i, j=1}^{n} a_{i j}^{\alpha \beta}(x) \xi_{i} \xi_{j} \leq \Lambda \omega_{\alpha \beta}(x)|\xi|^{2}$, for every $\xi \in \mathbb{R}$ and a.e. $x \in \Omega$, and $a_{i j}^{\alpha \beta}(x)=a_{j i}^{\alpha \beta}(x)$.

Proof. See [1], Lemma 3.1 or Lemma 4.13.

The following theorem will be proved in Section 3 .

Theorem 1.2. Suppose that

(H1) The function $g: \mathbb{R} \rightarrow \mathbb{R}$ is Lipschitz continuous (i.e., there exists a constant $C_{g}>0$ such that $\left|g\left(t_{1}\right)-g\left(t_{2}\right)\right| \leq C_{g}\left|t_{1}-t_{2}\right|$ for all $\left.t_{1}, t_{2} \in \mathbb{R}\right)$ and $g(0)=0$

(H2) $\omega \in A_{2}$;

(H3) $f_{j} / \omega \in L^{2}(\Omega, \omega), j=0,1, \ldots, n$;

(H4) The constant $\gamma=\lambda-C_{g}\left(C_{\Omega}^{2}+1\right)>0$ (with $C_{\Omega}$ as in Theorem 2.2). Then the problem $(P)$ has a unique solution $u \in W_{0}^{1,2}(\Omega, \omega)$ and there exists a constant $C>0$ such that

$$
\|u\|_{W_{0}^{1,2}(\Omega, \omega)} \leq C\left(\sum_{j=0}^{n}\left\|\frac{f_{j}}{\omega}\right\|_{L^{2}(\Omega, \omega)}\right) .
$$

Moreover, $u$ is the weak limit in $W_{0}^{1,2}\left(\Omega, \tilde{\omega}_{1}\right)$ of a sequence of solutions $u_{m} \in W_{0}^{1,2}\left(\Omega, \omega_{m}\right)$ of the problems

$$
\left(P_{m}\right)\left\{\begin{array}{l}
L_{m} u_{m}(x)+g\left(u_{m}(x)\right) \omega_{m}(x)=f_{0 m}(x)+\sum_{j=1}^{n} D_{j} f_{j m}(x) \text { in } \Omega, \\
u_{m}(x)=0 \text { on } \partial \Omega,
\end{array}\right.
$$

with $L_{m} u_{m}=-\sum_{i, j=1}^{n} D_{j}\left(a_{i j}^{m m}(x) D_{i} u_{m}(x)\right), f_{j m}=f_{j}\left(\omega_{m} / \omega\right)^{1 / 2}$ and $\omega_{m}=$ $\omega_{m m}$ (where $\omega_{m m}, a_{i j}^{m m}$ and $\tilde{\omega}_{1}$ are as Lemma 1.1).

2. Definitions and basic results. Let $\omega$ be a locally integrable nonnegative function in $\mathbb{R}^{n}$ and assume that $0<\omega(x)<\infty$ almost everywhere. We say that $\omega$ belongs to the Muckenhoupt class $A_{p}, 1<p<\infty$, or that $\omega$ 
is an $A_{p}$-weight, if there is a constant $C=C(p, \omega)$ such that

$$
\left(\frac{1}{|B|} \int_{B} \omega(x) d x\right)\left(\frac{1}{|B|} \int_{B} \omega^{1 /(1-p)}(x) d x\right)^{p-1} \leq C,
$$

for all balls $B \subset \mathbb{R}^{n}$, where $|$.$| denotes the n$-dimensional Lebesgue measure in $\mathbb{R}^{n}$. If $1<q \leq p$, then $A_{q} \subset A_{p}$ (see [4], [5] or [10] for more information about $A_{p}$-weights). The weight $\omega$ satisfies the doubling condition if there exists a positive constant $C$ such that $\mu(B(x ; 2 r)) \leq C \mu(B(x ; r))$ for every ball $B=B(x ; r) \subset \mathbb{R}^{n}$, where $\mu(B)=\int_{B} \omega(x) d x$. If $\omega \in A_{p}$, then $\mu$ is doubling (see Corollary 15.7 in [5]).

As an example of $A_{p}$-weight, the function $\omega(x)=|x|^{\alpha}, x \in \mathbb{R}^{n}$, is in $A_{p}$ if and only if $-n<\alpha<n(p-1)$ (see Corollary 4.4, Chapter IX in [9]).

If $\omega \in A_{p}$, then $\left(\frac{|E|}{|B|}\right)^{p} \leq C \frac{\mu(E)}{\mu(B)}$ whenever $B$ is a ball in $\mathbb{R}^{n}$ and $E$ is a measurable subset of $B$ (see 15.5 strong doubling property in [5]). Therefore, $\mu(E)=0$ if and only if $|E|=0$; so there is no need to specify the measure when using the ubiquitous expression almost everywhere and almost every, both abbreviated a.e.

Definition 2.1. Let $\omega$ be a weight, and let $\Omega \subset \mathbb{R}^{n}$ be open. For $0<p<\infty$ we define $L^{p}(\Omega, \omega)$ as the set of measurable functions $f$ on $\Omega$ such that

$$
\|f\|_{L^{p}(\Omega, \omega)}=\left(\int_{\Omega}|f(x)|^{p} \omega(x) d x\right)^{1 / p}<\infty .
$$

If $\omega \in A_{p}, 1<p<\infty$, then $\omega^{-1 /(p-1)}$ is locally integrable and we have $L^{p}(\Omega, \omega) \subset L_{\text {loc }}^{1}(\Omega)$ for every open set $\Omega$ (see Remark 1.2.4 in [10]). It thus makes sense to talk about weak derivatives of functions in $L^{p}(\Omega, \omega)$.

Definition 2.2. Let $\Omega \subset \mathbb{R}^{n}$ be open, and $\omega \in A_{2}$. We define the weighted Sobolev space $W^{1,2}(\Omega, \omega)$ as the set of functions $u \in L^{2}(\Omega, \omega)$ with weak derivatives $D_{j} u \in L^{2}(\Omega, \omega)$ for $j=1,2, \ldots, n$. The norm of $u$ in $W^{1,2}(\Omega, \omega)$ is defined by

$$
\|u\|_{W^{1,2}(\Omega, \omega)}=\left(\int_{\Omega}|u(x)|^{2} \omega(x) d x+\int_{\Omega}|\nabla u(x)|^{2} \omega(x) d x\right)^{1 / 2} .
$$

We also define $W_{0}^{1,2}(\Omega, \omega)$ as the closure of $C_{0}^{\infty}(\Omega)$ with respect to the norm (2.1).

If $\omega \in A_{2}$, then $W^{1,2}(\Omega, \omega)$ is the closure of $C^{\infty}(\Omega)$ with respect to the norm (2.1) (see Theorem 2.1.4 in [10]). The spaces $W^{1,2}(\Omega, \omega)$ and $W_{0}^{1,2}(\Omega, \omega)$ are Banach spaces.

It is evident that the weight function $\omega$ which satisfies $0<c_{1} \leq \omega(x) \leq c_{2}$ for $x \in \Omega$ ( $c_{1}$ and $c_{2}$ positive constants), gives nothing new (the space $\mathrm{W}_{0}^{1,2}(\Omega, \omega)$ is then identical with the classical Sobolev space $\left.\mathrm{W}_{0}^{1,2}(\Omega)\right)$. Consequently, we shall be interested above in all such weight functions $\omega$ which either vanish somewhere in $\Omega \cup \partial \Omega$ or increase to infinity (or both). 
The dual space of $W_{0}^{1,2}(\Omega, \omega)$ is the space

$$
\begin{aligned}
& {\left[W_{0}^{1,2}(\Omega, \omega)\right]^{*}=W^{-1,2}(\Omega, \omega)} \\
& =\left\{T=f_{0}-\operatorname{div} F: F=\left(f_{1}, \ldots, f_{n}\right), \frac{f_{j}}{\omega} \in L^{2}(\Omega, \omega), j=0, \ldots, n\right\},
\end{aligned}
$$

and $\|\cdot\|_{*}$ denotes the norm in $\left[W_{0}^{1,2}(\Omega, \omega)\right]^{*}$.

Definition 2.3. We say that an element $u \in W_{0}^{1,2}(\Omega, \omega)$ is weak solution of problem $(P)$ if

$$
\begin{aligned}
& \sum_{i, j=1}^{n} \int_{\Omega} a_{i j}(x) D_{i} u(x) D_{j} \varphi(x) d x+\int_{\Omega} g(u(x)) \omega(x) \varphi(x) d x \\
& =\int_{\Omega} f_{0}(x) \varphi(x) d x+\sum_{j=1}^{n} \int_{\Omega} f_{j}(x) D_{j} \varphi(x) d x
\end{aligned}
$$

for every $\varphi \in W_{0}^{1,2}(\Omega, \omega)$.

Remark 2.1. (a) If $A(x)=\left(a_{i j}(x)\right)$, we will use the notation

$$
\sum_{i, j=1}^{n} a_{i j}(x) D_{i} u(x) D_{j} \varphi(x)=(A(x) \nabla u(x)) \cdot \nabla \varphi(x),
$$

where the dot denotes the Euclidean scalar product in $\mathbb{R}^{n}$.

(b) Since the matrix $A(x)=\left(a_{i j}(x)\right)$ is symmetric, we have

$|(A(x) \nabla u(x)) \cdot \nabla \varphi(x)| \leq[(A(x) \nabla u(x)) \cdot \nabla u(x)]^{1 / 2}[(A(x) \nabla \varphi(x)) \cdot \nabla \varphi(x)]^{1 / 2}$.

Theorem 2.2 (The weighted Sobolev inequality). Let $\Omega$ be an open bounded set in $\mathbb{R}^{n}$ and $\omega \in A_{2}$. There exist positive constants $C_{\Omega}$ and $\delta$ such that for all $u \in W_{0}^{1,2}(\Omega, \omega)$ and all $\theta$ satisfying $1 \leq \theta \leq n /(n-1)+\delta$,

$$
\|u\|_{L^{2 \theta}(\Omega, \omega)} \leq C_{\Omega}\|\nabla u\|_{L^{2}(\Omega, \omega)} .
$$

Proof. It suffices to prove the inequality for functions $u \in C_{0}^{\infty}(\Omega)$ (see Theorem 1.3 in [2]). To extend the estimate (2.2) to arbitrary $u \in$ $W_{0}^{1,2}(\Omega, \omega)$, we let $\left\{u_{m}\right\}$ be a sequence of $C_{0}^{\infty}(\Omega)$ functions tending to $u$ in $W_{0}^{1,2}(\Omega, \omega)$. Applying the estimates $(2.2)$ to differences $u_{m_{1}}-u_{m_{2}}$, we see that $\left\{u_{m}\right\}$ will be a Cauchy sequence in $L^{2}(\Omega, \omega)$. Consequently, the limit function $u$ will lie in the desired spaces and satisfy (2.2).

Remark 2.3. By Theorem 2.2 (with $\theta=1$ ), we have

$$
\begin{aligned}
\|\nabla u\|_{L^{2}(\Omega, \omega)} & \leq\|u\|_{W_{0}^{1,2}(\Omega, \omega)}=\left(\int_{\Omega}|u|^{2} \omega d x+\int_{\Omega}|\nabla u|^{2} \omega d x\right)^{1 / 2} \\
& \leq\left(\left(C_{\Omega}^{2}+1\right) \int_{\Omega}|\nabla u|^{2} \omega d x\right)^{1 / 2}=C_{1}\|\nabla u\|_{L^{2}(\Omega, \omega)},
\end{aligned}
$$


where $C_{1}=\sqrt{C_{\Omega}^{2}+1}$.

3. Proof of Theorem 1.2. Part 1. Existence and uniqueness of solution.

The basic idea is to reduce the problem $(P)$ to an operator equation $\mathcal{A} u=T$ and apply the theorem below.

Theorem 3.1. Let $\mathcal{A}: X \rightarrow X^{*}$ be a monotone, coercive and hemicontinuous operator on the real, separable, reflexive Banach spaces $X$. Then the following assertions hold:

(a) for each $T \in X^{*}$ the equation $\mathcal{A} u=T$ has a solution $u \in X$;

(b) if the operator $\mathcal{A}$ is strictly monotone, then equation $\mathcal{A} u=T$ is uniquely solvable in $X$.

Proof. See Theorem 26.A in [11].

To prove Theorem 1.2, we define $B: W_{0}^{1,2}(\Omega, \omega) \times W_{0}^{1,2}(\Omega, \omega) \rightarrow \mathbb{R}$ and $T: W_{0}^{1,2}(\Omega, \omega) \rightarrow \mathbb{R}$ by

$$
\begin{aligned}
B(u, \varphi) & =\sum_{i, j=1}^{n} \int_{\Omega} a_{i j}(x) D_{i} u(x) D_{j} \varphi(x) d x+\int_{\Omega} g(u(x)) \varphi(x) \omega(x) d x \\
& =\int_{\Omega}(A(x) \nabla u(x)) \cdot \nabla \varphi(x) d x+\int_{\Omega} g(u(x)) \varphi(x) \omega(x) d x \\
T(\varphi) & =\int_{\Omega} f_{0}(x) \varphi(x) d x+\sum_{j=1}^{n} \int_{\Omega} f_{j}(x) D_{j} \varphi(x) d x .
\end{aligned}
$$

Step 1. By (H1) we have $|g(t)| \leq C_{g}|t|$. Using (1.2) and Remark 2.1 (b), we obtain

$$
\begin{aligned}
|B(u, \varphi)| \leq \int_{\Omega}|(A \nabla u) \cdot \nabla \varphi| d x+\int_{\Omega}|g(u) \| \varphi| \omega d x \\
\leq \int_{\Omega}((A \nabla u) \cdot \nabla u)^{1 / 2}((A \nabla \varphi) \cdot \nabla \varphi)^{1 / 2} d x+C_{g} \int_{\Omega}|u||\varphi| \omega d x \\
\leq\left(\int_{\Omega}(A \nabla u) \cdot \nabla u d x\right)^{1 / 2}\left(\int_{\Omega}(A \nabla \varphi) \cdot \nabla \varphi d x\right)^{1 / 2} d x \\
\quad+C_{g}\left(\int_{\Omega} u^{2} \omega d x\right)^{1 / 2}\left(\int_{\Omega} \varphi^{2} \omega d x\right)^{1 / 2} \\
\leq\left(\Lambda \int_{\Omega}|\nabla u|^{2} \omega d x\right)^{1 / 2}\left(\Lambda \int_{\Omega}|\nabla \varphi|^{2} \omega d x\right)^{1 / 2} \\
\quad+C_{g}\|u\|_{W_{0}^{1,2}(\Omega, \omega)}\|\varphi\|_{W_{0}^{1,2}(\Omega, \omega)} \\
\leq\left(\Lambda+C_{g}\right)\|u\|_{W_{0}^{1,2}(\Omega, \omega)}\|\varphi\|_{W_{0}^{1,2}(\Omega, \omega)},
\end{aligned}
$$


and by $(\mathrm{H} 3)$

$$
\begin{aligned}
|T(\varphi)| & \leq \int_{\Omega} \frac{\left|f_{0}\right|}{\omega}|\varphi| \omega d x+\sum_{j=1}^{n} \int_{\Omega} \frac{\left|f_{j}\right|}{\omega}\left|D_{j} \varphi\right| \omega d x \\
& \leq\left(\sum_{j=0}^{n}\left\|f_{j} / \omega\right\|_{L^{2}(\Omega, \omega)}\right)\|\varphi\|_{W_{0}^{1,2}(\Omega, \omega)} .
\end{aligned}
$$

Since $B(u,$.$) is linear, for each u \in W_{0}^{1,2}(\Omega, \omega)$, there is a linear continuous functional on $W_{0}^{1,2}(\Omega, \omega)$ denoted by $\mathcal{A} u$ such that $\langle\mathcal{A} u, \varphi\rangle=B(u, \varphi)$ for all $\varphi \in W_{0}^{1,2}(\Omega, \omega)$ (where $\langle f, x\rangle$ denotes the value of the functional $f$ at the point $x$ ). Moreover, by (3.1), we have

$$
\|\mathcal{A} u\|_{*} \leq\left(\Lambda+C_{g}\right)\|u\|_{W_{0}^{1,2}(\Omega, \omega)} .
$$

Hence, we obtain the operator

$$
\begin{aligned}
\mathcal{A}: W_{0}^{1,2}(\Omega, \omega) & \rightarrow\left[W_{0}^{1,2}(\Omega, \omega)\right]^{*} \\
u & \mapsto \mathcal{A} u .
\end{aligned}
$$

Consequently, problem $(P)$ is equivalent to the operator equation

$$
u \in W_{0}^{1,2}(\Omega, \omega): \mathcal{A} u=T .
$$

Step 2. The operator $\mathcal{A}$ is strictly monotone and coercive. In fact, if $u_{1}$, $u_{2} \in W_{0}^{1,2}(\Omega, \omega)$ we have, by (1.2) and Remark 2.3,

$$
\begin{aligned}
\left\langle\mathcal{A} u_{1}-\mathcal{A} u_{2}, u_{1}-u_{2}\right\rangle= & B\left(u_{1}, u_{1}-u_{2}\right)-B\left(u_{2}, u_{1}-u_{2}\right) \\
= & \int_{\Omega}\left(A \nabla\left(u_{1}-u_{2}\right)\right) \cdot \nabla\left(u_{1}-u_{2}\right) d x \\
& +\int_{\Omega}\left(g\left(u_{1}\right)-g\left(u_{2}\right)\right)\left(u_{1}-u_{2}\right) \omega d x \\
\geq & \lambda \int_{\Omega}\left|\nabla\left(u_{1}-u_{2}\right)\right|^{2} \omega d x-C_{g} \int_{\Omega}\left|u_{1}-u_{2}\right|^{2} \omega d x \\
\geq & \frac{\lambda}{C_{1}^{2}}\left\|u_{1}-u_{2}\right\|_{W_{0}^{1,2}(\Omega, \omega)}^{2}-C_{g}\left\|u_{1}-u_{2}\right\|_{W_{0}^{1,2}(\Omega, \omega)}^{2} \\
= & \beta\left\|u_{1}-u_{2}\right\|_{W_{0}^{1,2}(\Omega, \omega)}^{2},
\end{aligned}
$$

where $\beta=\frac{\lambda}{C_{1}^{2}}-C_{g}>0$. Therefore, the operator $\mathcal{A}$ is strongly monotone, and this implies that $\mathcal{A}$ is strictly monotone. Moreover, if $u \in W_{0}^{1,2}(\Omega, \omega)$, we have

$$
\begin{aligned}
\langle\mathcal{A} u, u\rangle & =B(u, u)=\int_{\Omega}(A \nabla u) \cdot \nabla u d x+\int_{\Omega} g(u) u \omega d x \\
& \geq \lambda \int_{\Omega}|\nabla u|^{2} \omega d x-C_{g} \int_{\Omega}|u|^{2} \omega d x
\end{aligned}
$$




$$
\begin{aligned}
& \geq \frac{\lambda}{C_{1}^{2}}\|u\|_{W_{0}^{1,2}(\Omega, \omega)}^{2}-C_{g}\|u\|_{W_{0}^{1,2}(\Omega, \omega)}^{2} \\
& \geq \beta\|u\|_{W_{0}^{1,2}(\Omega, \omega)}^{2} .
\end{aligned}
$$

Hence, $\frac{\langle\mathcal{A} u, u\rangle}{\|u\|_{W_{0}^{1,2}(\Omega, \omega)}} \rightarrow \infty$, as $\|u\|_{W_{0}^{1,2}(\Omega, \omega)} \rightarrow \infty$, that is, $\mathcal{A}$ is coercive.

Step 3. We need to show that the operator $\mathcal{A}$ is continuous. Let $u_{m} \rightarrow u$ in $W_{0}^{1,2}(\Omega, \omega)$. Then,

$$
\begin{aligned}
\left|B\left(u_{m}, \varphi\right)-B(u, \varphi)\right| & \leq \int_{\Omega}\left|\left(A \nabla\left(u_{m}-u\right)\right) \cdot \nabla \varphi\right| d x+\int_{\Omega}\left|g\left(u_{m}\right)-g(u)\right||\varphi| \omega d x \\
& \leq \Lambda\left(\int_{\Omega}\left|\nabla\left(u_{m}-u\right)\right|^{2} \omega d x\right)^{1 / 2}\left(\int_{\Omega}|\nabla \varphi|^{2} \omega d x\right)^{1 / 2} \\
& +C_{g} \int_{\Omega}\left|u_{m}-u \| \varphi\right| \omega d x \\
& \leq\left(\Lambda+C_{g}\right)\left\|u_{m}-u\right\|_{W_{0}^{1,2}(\Omega, \omega)}\|\varphi\|_{W_{0}^{1,2}(\Omega, \omega)},
\end{aligned}
$$

for all $\varphi \in W_{0}^{1,2}(\Omega, \omega)$. Then we obtain

$$
\left\|\mathcal{A} u_{m}-\mathcal{A} u\right\|_{*} \leq\left(\Lambda+C_{g}\right)\left\|u_{m}-u\right\|_{W_{0}^{1,2}(\Omega, \omega)} .
$$

Therefore, $\left\|\mathcal{A} u_{m}-\mathcal{A} u\right\|_{*} \rightarrow 0$ as $m \rightarrow \infty$. Hence, $\mathcal{A}$ is continuous and this implies that $\mathcal{A}$ is hemicontinuous.

By Theorem 3.1, the operator equation $\mathcal{A} u=T$ has unique solution $u \in W_{0}^{1,2}(\Omega, \omega)$ and it is the unique solution for problem $(P)$.

Part 2. Estimate for $\|u\|_{W_{0}^{1,2}(\Omega, \omega)}$.

In particular, for $\varphi=u$ in Definition 2.3, we have

$$
\begin{aligned}
& \sum_{i, j=1}^{n} \int_{\Omega} a_{i j}(x) D_{i} u(x) D_{j} u(x) d x+\int_{\Omega} g(u(x)) u(x) \omega(x) d x \\
& =\int_{\Omega} f_{0}(x) u(x) d x+\sum_{j=1}^{n} \int_{\Omega} f_{j}(x) D_{j} u(x) d x .
\end{aligned}
$$

(i) By (1.2) and Remark 2.3, we have

$$
\sum_{i, j=1}^{n} \int_{\Omega} a_{i j}(x) D_{i} u(x) D_{j} u(x) d x \geq \lambda \int_{\Omega}|\nabla u|^{2} \omega d x \geq \frac{\lambda}{C_{1}^{2}}\|u\|_{W_{0}^{1,2}(\Omega, \omega)}^{2},
$$

and by (H3) and Theorem 2.2 (with $\theta=1$ ),

$$
\begin{aligned}
\left|\int_{\Omega} f_{0} u d x\right| & \leq \int_{\Omega} \frac{\left|f_{0}\right|}{\omega}|u| \omega d x \\
& \leq\left\|f_{0} / \omega\right\|_{L^{2}(\Omega, \omega)}\|u\|_{L^{2}(\Omega, \omega)} \\
& \leq\left\|f_{0} / \omega\right\|_{L^{2}(\Omega, \omega)}\|u\|_{W_{0}^{1,2}(\Omega, \omega)}
\end{aligned}
$$


and analogously, for $j=1,2, \ldots, n$,

$$
\left|\int_{\Omega} f_{j} D_{j} u d x\right| \leq\left\|f_{j} / \omega\right\|_{L^{2}(\Omega, \omega)}\|u\|_{W_{0}^{1,2}(\Omega, \omega)} .
$$

(ii) By (H1), since $g(0)=0$, then $|g(t)| \leq C_{g}|t|$ for all $t \in \mathbb{R}$. By Theorem 2.2 (with $\theta=1$ ), we obtain

$$
\begin{aligned}
\left|\int_{\Omega} g(u) u \omega d x\right| & \leq \int_{\Omega}|g(u)||u| \omega d x \\
& \leq C_{g} \int_{\Omega}|u|^{2} \omega d x \\
& \leq C_{g}\|u\|_{W_{0}^{1,2}(\Omega, \omega)}^{2}
\end{aligned}
$$

Hence, in (3.3), we obtain

$$
\begin{aligned}
& \frac{\lambda}{C_{1}^{2}}\|u\|_{W_{0}^{1,2}(\Omega, \omega)}^{2}-C_{g}\|u\|_{W_{0}^{1,2}(\Omega, \omega)}^{2} \\
& \quad \leq\left(\sum_{j=0}^{n}\left\|f_{j} / \omega\right\|_{L^{2}(\Omega, \omega)}\right)\|u\|_{W_{0}^{1,2}(\Omega, \omega)} .
\end{aligned}
$$

Therefore,

$$
\|u\|_{W_{0}^{1,2}(\Omega, \omega)} \leq C\left(\sum_{j=0}^{n}\left\|f_{j} / \omega\right\|_{L^{2}(\Omega, \omega)}\right),
$$

where $C=C_{1}^{2} /\left(\lambda-C_{g} C_{1}^{2}\right)>0$.

Part 3. Approximation of solution.

Step 1. First, if $f_{j m}=f_{j}\left(\omega / \omega_{m}\right)^{-1 / 2}(j=0,1, \ldots, n)$, we note that

$$
\left\|\frac{f_{j m}}{\omega_{m}}\right\|_{L^{2}\left(\Omega, \omega_{m}\right)}=\left\|\frac{f_{j}}{\omega}\right\|_{L^{2}(\Omega, \omega)} .
$$

Then, if $u_{m} \in W_{0}^{1,2}\left(\Omega, \omega_{m}\right)$ is a solution of problem $\left(P_{m}\right)$, we have (by (3.4))

$$
\begin{aligned}
\left\|u_{m}\right\|_{W_{0}^{1,2}\left(\Omega, \omega_{m}\right)} & \leq C\left(\sum_{j=0}^{n}\left\|f_{j m} / \omega_{m}\right\|_{L^{2}\left(\Omega, \omega_{m}\right)}\right) \\
& =C\left(\sum_{j=0}^{n}\left\|f_{j} / \omega\right\|_{L^{2}(\Omega, \omega)}\right) \\
& =C_{3} .
\end{aligned}
$$

Using Lemma 1.1 and the inequality $\tilde{\omega}_{1} \leq \omega_{m}$, we obtain

$$
\left\|u_{m}\right\|_{W_{0}^{1,2}\left(\Omega, \tilde{\omega}_{1}\right)} \leq\left\|u_{m}\right\|_{W_{0}^{1,2}\left(\Omega, \omega_{m}\right)} \leq C_{3} .
$$


Consequently, $\left\{u_{m}\right\}$ is a bounded sequence in $W_{0}^{1,2}\left(\Omega, \tilde{\omega}_{1}\right)$. Therefore, there is a subsequence, again denoted by $\left\{u_{m}\right\}$, and $\tilde{u} \in W_{0}^{1,2}\left(\Omega, \tilde{\omega}_{1}\right)$ such that

$$
\begin{aligned}
& u_{m} \rightarrow \tilde{u} \text { in } L^{2}\left(\Omega, \tilde{\omega}_{1}\right), \\
& \left|\nabla u_{m}\right| \rightarrow|\nabla \tilde{u}| \text { in } L^{2}\left(\Omega, \tilde{\omega}_{1}\right), \\
& u_{m} \rightarrow \tilde{u} \text { a.e. in } \Omega,
\end{aligned}
$$

where the symbol " $\rightarrow$ " denotes weak convergence (see Theorem 1.31 in [5]).

Step 2. We have $\tilde{u} \in W_{0}^{1,2}(\Omega, \omega)$. In fact, for $F_{k}$ fixed, by (3.6) and (3.7), for all $\varphi \in W_{0}^{1,2}\left(\Omega, \tilde{\omega}_{1}\right)$, we obtain

$$
\begin{aligned}
& \int_{\Omega} u_{m} \varphi \tilde{\omega}_{1} d x \rightarrow \int_{\Omega} \tilde{u} \varphi \tilde{\omega}_{1} d x, \\
& \int_{\Omega} D_{i} u_{m} D_{i} \varphi \tilde{\omega}_{1} d x \rightarrow \int_{\Omega} D_{i} \tilde{u} D_{i} \varphi \tilde{\omega}_{1} d x .
\end{aligned}
$$

If $\psi \in W_{0}^{1,2}(\Omega, \omega)$, then $\varphi=\psi \chi_{F_{k}} \in W_{0}^{1,2}\left(\Omega, \tilde{\omega}_{1}\right)$ (since $\omega \sim \tilde{\omega}_{1}$ in $F_{k}$, i.e., there is a constant $c>0$ such that $\tilde{\omega}_{1} \leq c \omega$ in $F_{k}$, and $\chi_{E}$ denotes the characteristic function of a measurable set $E \subset \mathbb{R}^{n}$ ) and

$$
\begin{aligned}
& \int_{\Omega} \varphi^{2} \tilde{\omega}_{1} d x=\int_{F_{k}} \psi^{2} \tilde{\omega}_{1} d x \leq c \int_{F_{k}} \psi^{2} \omega d x \leq c \int_{\Omega} \psi^{2} \omega d x<\infty \\
& \int_{\Omega}\left(D_{i} \varphi\right)^{2} \tilde{\omega}_{1} d x=\int_{F_{k}}\left(D_{i} \psi\right)^{2} \tilde{\omega}_{1} d x \leq c \int_{F_{k}}\left(D_{i} \psi\right)^{2} \omega d x \leq c \int_{\Omega}\left(D_{i} \psi\right)^{2} \omega d x<\infty .
\end{aligned}
$$

Consequently,

$$
\begin{aligned}
& \int_{\Omega} u_{m} \psi \chi_{F_{k}} \tilde{\omega}_{1} d x \rightarrow \int_{\Omega} \tilde{u} \psi \chi_{F_{k}} \tilde{\omega}_{1} d x, \\
& \int_{\Omega} D_{i} u_{m} D_{i} \psi \chi_{F_{k}} \tilde{\omega}_{1} d x \rightarrow \int_{\Omega} D_{i} \tilde{u} D_{i} \psi \chi_{F_{k}} \tilde{\omega}_{1} d x,
\end{aligned}
$$

for all $\psi \in W_{0}^{1,2}(\Omega, \omega)$, that is, the sequence $\left\{u_{m} \chi_{F_{k}}\right\}$ is weakly convergent in $W_{0}^{1,2}(\Omega, \omega)$. Therefore, we have

$$
\|\nabla \tilde{u}\|_{L^{2}\left(F_{k}, \omega\right)}^{2}=\int_{F_{k}}|\nabla \tilde{u}|^{2} \omega d x \leq \limsup _{m \rightarrow \infty} \int_{F_{k}}\left|\nabla u_{m}\right|^{2} \omega d x,
$$

and for $m \geq k$, we have $\omega=\omega_{m}$ in $F_{k}$. Hence, by (3.5), we obtain

$$
\begin{aligned}
\|\nabla \tilde{u}\|_{L^{2}\left(F_{k}, \omega\right)}^{2} & \leq \limsup _{m \rightarrow \infty} \int_{F_{k}}\left|\nabla u_{m}\right|^{2} \omega d x=\limsup _{m \rightarrow \infty} \int_{F_{k}}\left|\nabla u_{m}\right|^{2} \omega_{m} d x \\
& \leq \limsup _{m \rightarrow \infty} \int_{\Omega}\left|\nabla u_{m}\right|^{2} \omega_{m} d x \leq C_{3}^{2} .
\end{aligned}
$$

By the Monotone Convergence Theorem, we obtain $\|\nabla \tilde{u}\|_{L^{2}(\Omega, \omega)} \leq C_{3}$. Therefore, we have $\tilde{u} \in W_{0}^{1,2}(\Omega, \omega)$. 
Step 3. We need to show that $\tilde{u}$ is a solution of problem $(P)$, i.e, for every $\varphi \in W_{0}^{1,2}(\Omega, \omega)$ we have

$$
\begin{aligned}
& \sum_{i, j=1}^{n} \int_{\Omega} a_{i j}(x) D_{i} \tilde{u}(x) D_{j} \varphi(x) d x+\int_{\Omega} g(\tilde{u}(x)) \varphi(x) \omega(x) d x \\
& =\int_{\Omega} f_{0}(x) \varphi(x) d x+\sum_{j=1}^{n} \int_{\Omega} f_{j}(x) D_{j} \varphi(x) d x .
\end{aligned}
$$

Using the fact that $u_{m}$ is a solution of $\left(P_{m}\right)$, we have

$$
\begin{aligned}
& \sum_{i, j=1}^{n} \int_{\Omega} a_{i j}^{m m}(x) D_{i} u_{m}(x) D_{j} \varphi(x) d x+\int_{\Omega} g\left(u_{m}(x)\right) \varphi(x) \omega_{m}(x) d x \\
& =\int_{\Omega} f_{m}(x) \varphi(x) d x+\sum_{j=1}^{n} \int_{\Omega} f_{j m}(x) D_{j} \varphi(x) d x
\end{aligned}
$$

for every $\varphi \in W_{0}^{1,2}\left(\Omega, \omega_{m}\right)$. Moreover, over $F_{k}$ (for $m \geq k$ ) we have the following properties:

(i) $\omega=\omega_{m}$;

(ii) $f_{j m}=f_{j}, j=0,1,2 \ldots, n$;

(iii) $a_{i j}^{m m}(x)=a_{i j}(x)$.

For $\varphi \in W_{0}^{1,2}(\Omega, \omega)$ and $k>0$ (fixed), we define $G_{1}, G_{2}: W_{0}^{1,2}\left(\Omega, \tilde{\omega}_{1}\right) \rightarrow \mathbb{R}$ by

$$
\begin{aligned}
& G_{1}(u)=\sum_{i, j=1}^{n} \int_{\Omega} a_{i j}(x) D_{i} u(x) D_{j} \varphi(x) \chi_{F_{k}}(x) d x, \\
& G_{2}(u)=\int_{\Omega} g(u(x)) \varphi(x) \omega(x) \chi_{F_{k}}(x) d x .
\end{aligned}
$$

(a) We see that the functional $G_{1}$ is linear and continuous. In fact, we have (by Lemma 1.1 (iv)) $\omega \sim \tilde{\omega}_{1}$ in $F_{k}\left(\omega \leq c \tilde{\omega}_{1}\right)$. By (2), we obtain

$$
\begin{aligned}
\left|G_{1}(u)\right| & \leq \int_{F_{k}}|(A \nabla u) \cdot \nabla \varphi| d x \leq \int_{F_{k}}((A \nabla u) \cdot \nabla u)^{1 / 2}((A \nabla \varphi) \cdot \nabla \varphi)^{1 / 2} d x \\
& \left.\leq\left(\int_{F_{k}}(A \nabla u) \cdot \nabla u d x\right)^{1 / 2}\left(\int_{F_{k}}(A \nabla \varphi) \cdot \nabla \varphi\right)^{1 / 2} d x\right)^{1 / 2} \\
& \leq \Lambda\left(\int_{F_{k}}|\nabla u|^{2} \omega d x\right)^{1 / 2}\left(\int_{F_{k}}|\nabla \varphi|^{2} \omega d x\right)^{1 / 2} \\
& \leq \Lambda\left(\int_{F_{k}} c|\nabla u|^{2} \tilde{\omega}_{1} d x\right)^{1 / 2}\left(\int_{\Omega}|\nabla \varphi|^{2} \omega d x\right)^{1 / 2} \\
& \leq \Lambda c^{1 / 2}\|\varphi\|_{W_{0}^{1,2}(\Omega, \omega)}\|u\|_{W_{0}^{1,2}\left(\Omega, \tilde{\omega}_{1}\right)}
\end{aligned}
$$


(b) We see that $G_{2}$ is a continuous functional. In fact, if $u_{1}, u_{2} \in W_{0}^{1,2}\left(\Omega, \tilde{\omega}_{1}\right)$, we obtain by $(\mathrm{H} 1)$

$$
\begin{aligned}
\left|G_{2}\left(u_{2}\right)-G_{2}\left(u_{1}\right)\right| & \leq \int_{F_{k}}\left|g\left(u_{2}\right)-g\left(u_{1}\right)\right||\varphi| \omega d x \\
& \leq \int_{F_{k}} C_{g}\left|u_{1}-u_{2}\right||\varphi| \omega d x \\
& \leq C_{g}\left(\int_{F_{k}}|\varphi|^{2} \omega d x\right)^{1 / 2}\left(\int_{F_{k}}\left|u_{1}-u_{2}\right|^{2} \omega d x\right)^{1 / 2} \\
& \leq C_{g}\left(\int_{\Omega}|\varphi|^{2} \omega d x\right)^{1 / 2}\left(\int_{F_{k}} c\left|u_{1}-u_{2}\right|^{2} \tilde{\omega}_{1} d x\right)^{1 / 2} \\
& \leq c^{1 / 2} C_{g}\|\varphi\|_{W_{0}^{1,2}(\Omega, \omega)}\left\|u_{1}-u_{2}\right\|_{W_{0}^{1,2}\left(\Omega, \tilde{\omega}_{1}\right)} .
\end{aligned}
$$

Using (a), (b), properties (i), (ii) and (iii), and basing on the fact that $u_{m}$ is the solution of $\left(P_{m}\right)$, we obtain

$$
\begin{aligned}
& \sum_{i, j=1}^{n} \int_{F_{k}} a_{i j}(x) D_{i} \tilde{u}(x) D_{j} \varphi(x) d x+\int_{F_{k}} g(\tilde{u}(x)) \varphi(x) \omega(x) d x \\
= & \lim _{m \rightarrow \infty}\left[G_{1}\left(u_{m}\right)+G_{2}\left(u_{m}\right)\right] \\
= & \lim _{m \rightarrow \infty}\left(\sum_{i, j=1}^{n} \int_{F_{k}} a_{i j}^{m m}(x) D_{i} u_{m}(x) D_{j} \varphi(x) d x+\int_{F_{k}} g\left(u_{m}(x)\right) \varphi(x) \omega_{m}(x) d x\right) \\
= & \lim _{m \rightarrow \infty}\left(\sum_{i, j=1}^{n} \int_{\Omega} a_{i j}^{m m}(x) D_{i} u_{m}(x) D_{j} \varphi(x) d x+\int_{\Omega} g\left(u_{m}(x)\right) \varphi(x) \omega_{m}(x) d x\right. \\
& \left.-\sum_{i, j=1}^{n} \int_{\Omega \cap F_{k}^{c}} a_{i j}^{m m}(x) D_{i} u_{m}(x) D_{j} \varphi(x) d x-\int_{\Omega \cap F_{k}^{c}} g\left(u_{m}(x)\right) \varphi(x) \omega_{m}(x) d x\right) \\
= & \lim _{m \rightarrow \infty}\left(\int_{\Omega} f_{0 m}(x) \varphi(x) d x+\sum_{j=1}^{n} \int_{\Omega} f_{j m}(x) D_{j} \varphi(x) d x\right. \\
& \left.-\sum_{i, j=1}^{n} \int_{\Omega \cap F_{k}^{c}} a_{i j}^{m m}(x) D_{i} u_{m}(x) D_{j} \varphi(x) d x-\int_{\Omega \cap F_{k}^{c}} g\left(u_{m}(x)\right) \varphi(x) \omega_{m}(x) d x\right),
\end{aligned}
$$

where $E^{c}$ denotes the complement of a set $E \subset \mathbb{R}^{n}$.

(I) By the Lebesgue Dominated Convergence Theorem and $\tilde{\omega}_{2} \in A_{2}$, we obtain $($ as $m \rightarrow \infty)$

$$
\begin{aligned}
& \int_{\Omega} f_{m} \varphi d x \rightarrow \int_{\Omega} f \varphi d x \\
& \int_{\Omega} f_{j m} D_{j} \varphi d x \rightarrow \int_{\Omega} f_{j} D_{j} \varphi d x,(j=1, \ldots, n) .
\end{aligned}
$$


(II) Since the matrix $A^{m}(x)=\left(a_{i j}^{m m}\right)(x)$ is symmetric, we have

$$
\left|\left(A^{m} \nabla u_{m}\right) \cdot \nabla \varphi\right| \leq\left[\left(A^{m} \nabla u_{m}\right) \cdot \nabla u_{m}\right]^{1 / 2}\left[\left(A^{m} \nabla \varphi\right) \cdot \nabla \varphi\right]^{1 / 2} .
$$

Then, by Lemma 1.1 (vi) and (3.5), we obtain

$$
\begin{aligned}
& \left|\sum_{i, j=1}^{n} \int_{\Omega \cap F_{k}^{c}} a_{i j}^{m m} D_{i} u_{m} D_{j} \varphi d x\right| \leq \int_{\Omega \cap F_{k}^{c}}\left|\left(A^{m} \nabla u_{m}\right) \cdot \nabla \varphi\right| d x \\
& \quad \leq \Lambda\left(\int_{\Omega \cap F_{k}^{c}}\left|\nabla u_{m}\right|^{2} \omega_{m} d x\right)^{1 / 2}\left(\int_{\Omega \cap F_{k}^{c}}|\nabla \varphi|^{2} \omega_{m} d x\right)^{1 / 2} \\
& \quad \leq \Lambda\left\|u_{m}\right\|_{W_{0}^{1,2}\left(\Omega, \omega_{m}\right)}\left(\int_{\Omega \cap F_{k}^{c}}|\nabla \varphi|^{2} w_{m} d x\right)^{1 / 2} \\
& \quad \leq \Lambda C_{3}\left(\int_{\Omega \cap F_{k}^{c}}|\nabla \varphi|^{2} w_{m} d x\right)^{1 / 2} .
\end{aligned}
$$

(III) By (H1), $|g(t)| \leq C_{g}|t|$ (for all $t \in \mathbb{R}$ ), and (3.5), we have

$$
\begin{aligned}
& \left|\int_{\Omega \cap F_{k}^{c}} g\left(u_{m}\right) \varphi \omega_{m} d x\right| \leq \int_{\Omega \cap F_{k}^{c}}\left|g\left(u_{m}\right)\right||\varphi| \omega_{m} d x \\
& \quad \leq C_{g} \int_{\Omega \cap F_{k}^{c}}\left|u_{m}\right||\varphi| \omega_{m} d x \\
& \quad \leq C_{g}\left(\int_{\Omega \cap F_{k}^{c}}\left|u_{m}\right|^{2} \omega_{m} d x\right)^{1 / 2}\left(\int_{\Omega \cap F_{k}^{c}}|\varphi|^{2} \omega_{m} d x\right)^{1 / 2} \\
& \quad \leq C_{g}\left\|u_{m}\right\|_{W_{0}^{1,2}\left(\Omega, \omega_{m}\right)}\left(\int_{\Omega \cap F_{k}^{c}}|\varphi|^{2} \omega_{m} d x\right)^{1 / 2} \\
& \quad \leq C_{g} C_{3}\left(\int_{\Omega \cap F_{k}^{c}}|\varphi|^{2} \omega_{m} d x\right)^{1 / 2} .
\end{aligned}
$$

Using Lemma 1.1, we know that $\left|\Omega \cap F_{k}^{c}\right| \rightarrow 0$ when $k \rightarrow \infty$. Then

$$
\lim _{k \rightarrow \infty}\left(\int_{\Omega \cap F_{k}^{c}}|\varphi|^{2} \omega_{m} d x\right)^{1 / 2}=\lim _{k \rightarrow \infty}\left(\int_{\Omega \cap F_{k}^{c}}|\nabla \varphi|^{2} \omega_{m} d x\right)^{1 / 2}=0
$$

and we obtain in (3.9) and (3.10)

$$
\begin{aligned}
& \lim _{k \rightarrow \infty} \sum_{i, j=1}^{n} \int_{\Omega \cap F_{k}^{c}} a_{i j}^{m m}(x) D_{i} u_{m}(x) D_{j} \varphi(x) d x=0, \\
& \lim _{k \rightarrow \infty} \int_{\Omega \cap F_{k}^{c}} g\left(u_{m}\right) \varphi \omega_{m} d x=0 .
\end{aligned}
$$


Therefore, by (3.7), (3.11) and (3.12) we conclude, when $k \rightarrow \infty$ (and $m \geq k)$,

$$
\sum_{i, j=1}^{n} \int_{\Omega} a_{i j} D_{i} \tilde{u} D_{j} \varphi d x+\int_{\Omega} g(\tilde{u}) \varphi \omega d x=\int_{\Omega} f_{0} \varphi d x+\sum_{j=1}^{n} \int_{\Omega} f_{j} D_{j} \varphi d x,
$$

for all $\varphi \in W_{0}^{1,2}(\Omega, \omega)$, that is, $\tilde{u}$ is a solution of the problem $(P)$. Therefore, $u=\tilde{u}$ (by the uniqueness).

Example 1. Let $\Omega=\left\{(x, y) \in \mathbb{R}^{2}: x^{2}+y^{2}<1\right\}$ and $0<C_{\Omega}^{2}+1<$ $a<b$. By Theorem 1.2, with $g(t)=\sin (t)$ (with $C_{g}=1$ ), $f_{0}(x, y)=x|y|$, $f_{1}(x, y)=|x| y \cos (x y), f_{2}(x, y)=|x| y \sin (x y), \omega(x, y)=\left(x^{2}+y^{2}\right)^{-1 / 2}$ and

$$
\mathcal{A}(x, y)=\left(\begin{array}{cc}
a\left(x^{2}+y^{2}\right)^{-1 / 2} & 0 \\
0 & b\left(x^{2}+y^{2}\right)^{-1 / 2}
\end{array}\right),
$$

the problem

$$
\left\{\begin{array}{l}
L u(x, y)+g(u(x, y)) \omega(x, y)(x)=f_{0}(x, y)-\frac{\partial f_{1}}{\partial x}(x, y)-\frac{\partial f_{2}}{\partial y}(x, y) \text { in } \Omega \\
u(x)=0 \text { on } \partial \Omega
\end{array}\right.
$$

where

$$
L u(x)=-\frac{\partial}{\partial x}\left(a\left(x^{2}+y^{2}\right)^{-1 / 2} \frac{\partial u}{\partial x}\right)-\frac{\partial y}{\partial x}\left(b\left(x^{2}+y^{2}\right)^{-1 / 2} \frac{\partial u}{\partial y}\right)
$$

has a unique solution $u \in W_{0}^{1,2}(\Omega, \omega)$ and $u$ can be approximated by a sequence of solutions for non-degenerate nonlinear elliptic equations.

\section{REFERENCES}

[1] Cavalheiro, A. C., An approximation theorem for solutions of degenerate elliptic equations, Proc. Edinb. Math. Soc. 45 (2002), 363-389.

[2] Fabes, E., Kenig, C., Serapioni, R., The local regularity of solutions of degenerate elliptic equations, Comm. Partial Differential Equations 7 (1982), 77-116.

[3] Fernandes, J. C., Franchi, B., Existence and properties of the Green function for a class of degenerate parabolic equations, Rev. Mat. Iberoam. 12 (1996), 491-525.

[4] García-Cuerva, J., Rubio de Francia, J. L., Weighted Norm Inequalities and Related Topics, North-Holland Publishing Co., Amsterdam, 1985.

[5] Heinonen, J., Kilpeläinen, T., Martio, O., Nonlinear Potential Theory of Degenerate Elliptic Equations, Oxford University Press, Oxford, 1993.

[6] Kufner, A., Weighted Sobolev Spaces, John Wiley \& Sons, New York, 1985.

[7] Muckenhoupt, B., Weighted norm inequalities for the Hardy maximal function, Trans. Amer. Math. Soc. 165 (1972), 207-226.

[8] Murthy, M. K. V., Stampacchia, G., Boundary value problems for some degenerate elliptic operators, Ann. Mat. Pura Appl. 80 (1) (1968), 1-122.

[9] Torchinsky, A., Real-Variable Methods in Harmonic Analysis, Academic Press, San Diego, 1986.

[10] Turesson, B. O., Nonlinear Potential Theory and Weighted Sobolev Spaces, SpringerVerlag, Berlin, 2000. 
[11] Zeidler, E., Nonlinear Functional Analysis and Its Applications. Vol. II/B, SpringerVerlag, New York, 1990.

Albo Carlos Cavalheiro

Department of Mathematics

State University of Londrina

Londrina - PR - Brazil, 86057-970

e-mail: accava@gmail.com

Received June 27, 2017 